\title{
THE IMPORTANCE OF CONCEPT AND DESIGN VISUALISATION IN THE PRODUCTION OF AN AUTOMATED ASSEMBLY AND TEST MACHINE
}

\author{
Daniel Smale BEng (Hons) AMIMechE \\ TQC Ltd, Hooton Street, Nottingham, NG3 2NJ, UK, daniel.smale@tqc.ca.uk
}

Abstract: Producing an automated assembly and test machine is a lengthy process consisting of numerous stages. It can involve several iterations through some or, very occasionally, all of these stages. There is one major similarity linking these stages: the requirement for consistent and accurate information. This unifying requirement makes communication a crucial, but often overlooked, aspect of producing an assembly automation machine.

It is often the case that the simplest and most reliable method of communication is a visual representation or "Visualisation". Visualisation can and does take many forms; anything from sketches on napkins to detailed 3D computer images. All formats have benefits as well as drawbacks.

This paper looks at the role of visualisation and its importance within the production of an automated assembly and test machine. It also further develops the issues and problems faced by the assembly automation industry as well as looking in greater detail at the potential solutions and how these are being developed and expanded on by European research projects.

Key words: Communication, design, visualisation. 


\section{INTRODUCTION}

In the 21 st century, communication is affordable, instant and omnipresent. It is because of this that it is easy to forget how essential it can be. Not simply in terms of the physical (or electronic) actions, but rather in terms of the nature of these actions. Everyone is aware of the saying "a picture is worth a thousand words..." something which is probably true except that, in many cases, it under-sells the value of visual representations. Drawings, be they sketches, diagrams or full engineering detailed views, can convey that which words often cannot. This is increasingly the case as international projects become more common where linguistic barriers can result in confusion.

The marketplace today is heavily consumer driven. The increasing consumer expectation and product competition places great pressures on the producers, and this pressure is felt through the whole supply line. A system integrator is generally somewhere in the middle of this line and so feels this pressure not only from the customer who wants his or her new product to be manufactured, assembled and tested as soon as possible, but also from the suppliers who struggle to meet strict lead times and increasing performance expectations.

In this climate, constant and good quality communication is essential for any project to be a success. Visualisation is the engineer's favoured means of communication and thus, as the pressure on the engineers increases, so it does on the visualisation.

\section{ROLES AND IMPORTANCE}

Engineers have not failed in recognising the value of images; that is why engineering drawings exist. A good engineer tends to be one who can convey ideas and concepts in visual representations, not necessarily one who is good with words, though it is often of benefit to be capable of both. Visualisations reduce the need for complex verbal communications, which are subject to misinterpretation and distortion over time; people generally remember different sequences and outcomes from conversations and meetings. Visualisations, at least good ones, do not allow room for misinterpretation and are traceable. 
Meetings and conversations rely upon human interaction and are thus inherently social situations. As a consequence, there is the constant possibility that the ultimate objectives will break down to mundane interactions [1,2]. Visualisations can often be a means of directing conversations and offer a plainly visible objective.

Visualisation has the main purpose of communicating concepts, ideas and detailed information concerning all aspects of the system. This single purpose can be divided into two distinct roles: Inter-company and Intracompany.

Inter-company communication occurs between two or more companies involved in the supply chain. This is where the biggest gains and, by inference, losses can be made.

Intra-company communication occurs internally within a single company and does not involve any outside personnel. Intra-company is much more frequent and generally less formalised than Inter-company communication and so is less crucial for the communication to be right first time as there are more opportunities for corrections. This effect is lessened as the company becomes larger to the extent that, in the case of large multinationals, Intracompany communication only really applies to one department or branch.

\section{FORMS OF VISUALISATION - FOUR CLASSIFICATIONS}

There are many different types or forms of visualisation. These could be classified as follows: Sketch, Diagram, Drawing, and Detail. It should be noted that these classes are independent of media, (i.e. just because a visualisation is hand-drawn does not automatically mean that it is a Sketch) and also independent of the purpose, or role, of the visualisation.

The above classification list is shown in ascending order of level of detail, degree of prescription and in time to produce (and thus also in ascending order of cost to alter). It is additionally in descending order of the need for additional communication, i.e. a Sketch is often most effective when used during a conversation whereas a Detail contains all the relevant 
information needed for manufacture and therefore can be seen as "standalone".

It should be noted that, in the following definitions, the intended "audience" or receivers of the information are assumed to have some engineering experience or understanding.

A Sketch can be defined as a visualisation that is used to assist verbal communication, not to replace it. A Sketch will generally be produced "adhoc" and will not be prepared prior to a meeting or conversation. It should be very quick to produce, not dimensionally accurate or even scaled appropriately. Finally, the level of detail should be the minimum necessary to convey the concept to the other person or people involved.

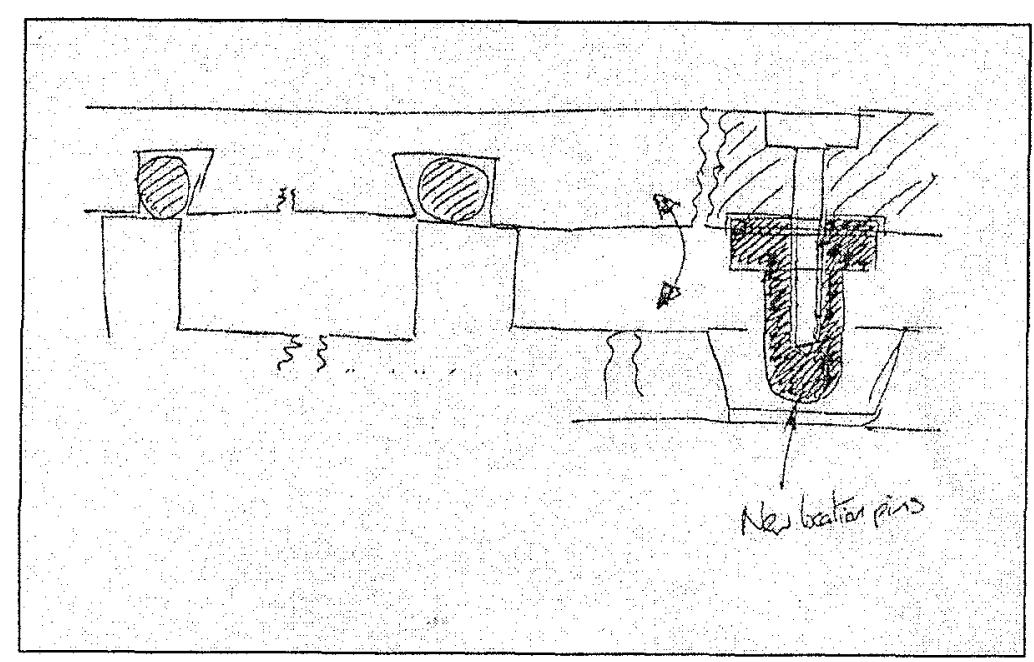

Figure 1. An example of a Sketch.

A Diagram, essentially, can be defined as a visualisation that is more detailed, accurate and time-consuming to produce than a Sketch. However, a more accurate definition would be that a Diagram is also used to assist in verbal communication but, unlike a Sketch, it is generally prepared in advance of any meeting or conversation. It is likely to consist of a series of boxes which, though approximately correct in scale and dimensions, will generally require labelling so that their nature or purpose is clear. 


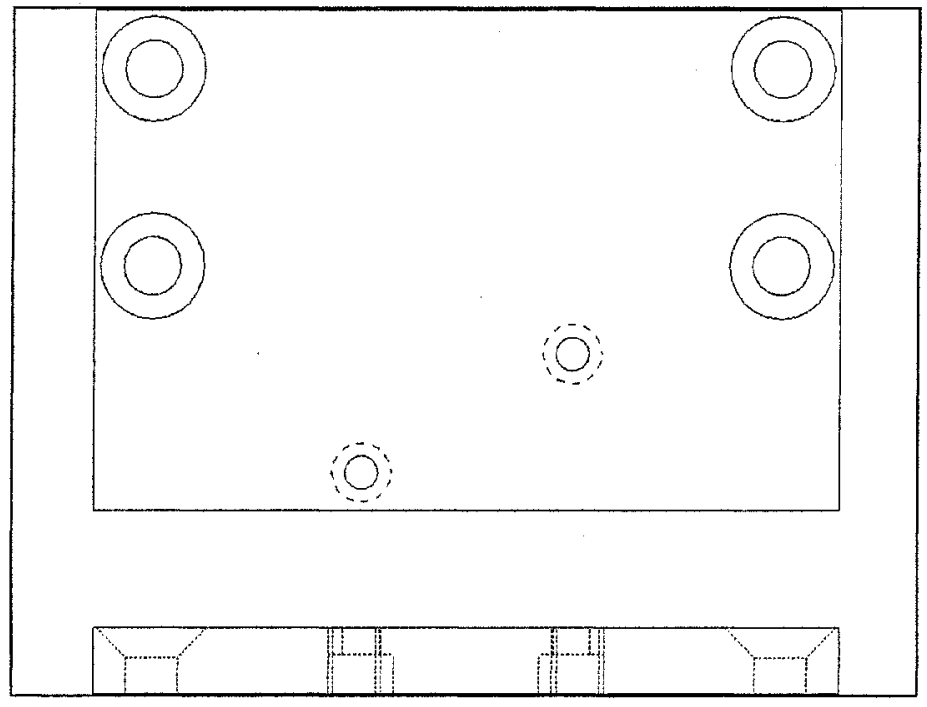

Figure 2. An example of a Diagram.

The next most descriptive visualisation is the Drawing. This can be defined as a representation that is accurate in both dimensions and scaling. It will be able to accurately convey the product or system in terms of layout, functionality and purpose. The visualisation should require minimal additional communication to be fully understood, though it may be presented as part of a meeting or conversation. The relativity of parts and components to one-another should be clearly visible. An example of this classification could often be the "GA" or General Assembly drawing. 


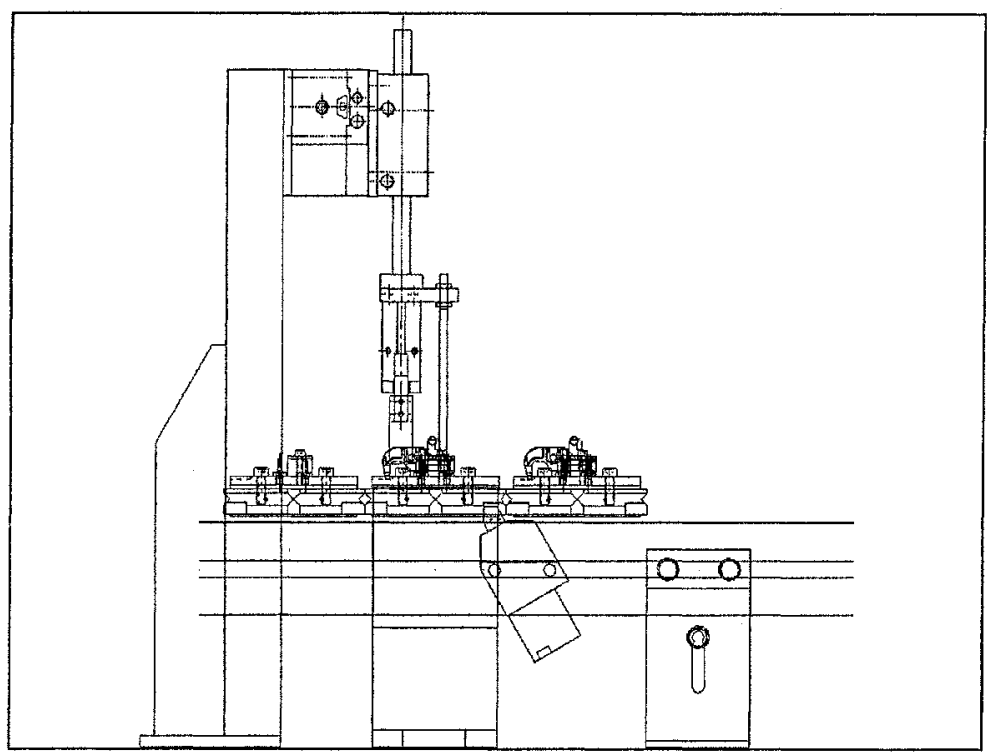

Figure 3. An example of a Drawing. Courtesy of Southco and TQC Ltd.

Finally; the definition of a Detail. This final classification is, as the name suggests, the most detailed and therefore most time-consuming and costly to produce. The visualisation should contain every piece of information needed for manufacture and/or assembly. It should therefore be entirely selfsupporting and not require any commentary: it replaces verbal communication. 


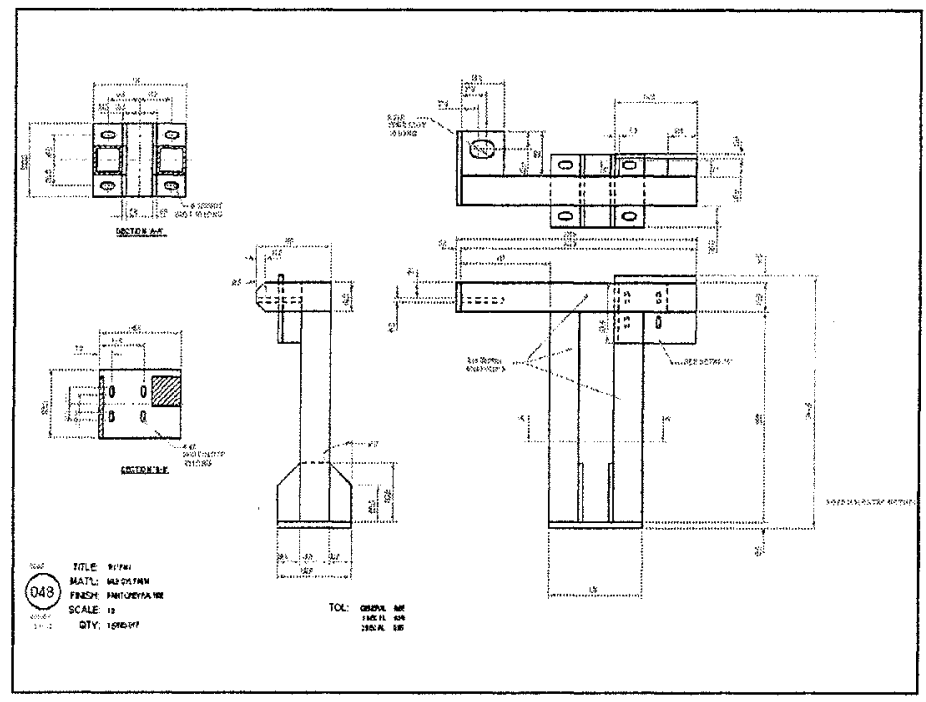

Figure 4. An example of a Detail. Courtesy of Southco and TQC Ltd.

Naturally, there are no clear lines that distinguish between the different classifications. However, most visualisations will fall more into one definition than the others.

Any engineering project will involve all of the previously mentioned classes of communication. The class used will vary depending on the phase of the project, though it must be noted that the project will go through numerous iterations of these phases. The class will also depend, to a lesser extent, on between whom the communication is occurring and their geographical proximity.

With regard to the project phase, during the early stages (conceptualisation) communication will generally be in the Sketch class. This is because whilst there are several concepts, and thus potential paths for the project team, there is no point in wasting time producing Details. This is often aided by the team members working in the same office, or otherwise having frequent physical meetings

As the project progresses in time, and hopefully through the design phases, it is necessary to make decisions that will reduce the number of 
potential solutions. This is a very iterative process and will generally entail investigating some or all of the options through discussion and the use of Diagrams. In modern projects, where system lead times are often measured in weeks, this process can be performed in a matter of a few days, when a "final" decision regarding the system design is made.

It is usually at this point that the team will progress to using Drawings to finalise the assembly and test process and system layout/configuration. Subject to a final review, the team will then move to Details that can then be used for production of the complete system.

Regarding the effects of who is involved in the communication and their geographical locations; the frequency of inter- and intra-company communication is significantly different. Generally, this frequency is related to the distance between those involved.

Additionally, it is usually the case that the progression through the class of visualisations are over a different timescale, have different start points and are out of phase with one-another. This in itself can be for a number of reasons that will not be covered in this paper.

\section{PROBLEMS}

The Inter-company communication occurs less frequently than Intracompany communication. For this reason, Inter-company meetings are more structured and there is considerable pressure for them to go well first time, driven by the time and monetary costs associated with them. It is therefore desirable to maximise the relevant detail that can be conveyed. However, it is also desirable to minimise the complexity of the visualisation. This is for two reasons: Firstly, complexity generally requires a trained eye to understand. Secondly, complexity is time-consuming to produce, which in turn is an additional cost that can be ill afforded. Both of these problems are expanded upon below.

The fact that engineering drawings require a trained eye to fully understand them has not been an issue whilst the decisions being made regarding the assembly machine and the associated project were conducted, at least predominantly, by engineers within the company.

This is, however, changing. These decisions are not confined simply to the initial sell of the design to a customer; management and financial 
considerations come into play at every step, both internally within the company itself and externally. This change has been catalysed by the evermore competitive market and the subsequent increasing importance of all cost issues. Assembly and test automation machines are usually a very large investment and so, quite understandably, the management of the customer company want to ensure that the investment is financially sound.

The management will not only base their decision on financial matters, the overall business strategy of the company will almost certainly play a role. It is therefore important, from all stakeholders' perspectives, that staff without an engineering background can understand the ideas, concepts and designs.

Equally, the quoting process itself, from the system integrator to the customer and also from equipment suppliers to the system integrator, can be an expensive one to undertake. The current economic climate, as described in the Introduction, forces each member of the supply chain to be efficient and minimise wastage. No company can afford to repeatedly spend tens or even hundreds of hours preparing quotes and in other sales activities, which do not directly result in a return on this investment. However, the dilemma is that without spending this time and effort, there is little chance of securing any business.

The other issue associated with engineering drawings is that they are time consuming to produce. This is especially the case with CAD modelling, which is often the most time-consuming part of the whole process [3]. The acceleration of product evolution, which is driven by several factors, has reduced the time span of product lifecycles. This has impacted upon the design and manufacture aspects of the product. Neither the individual assembly automation projects nor the companies themselves can afford to waste time producing detailed drawings that, for one reason or another, will not be used. The current way of working amongst all of the stakeholders is to continually monitor and balance this work depending on the situation. This requires continual human decision-making and thus, by implication, mistakes are made. These are rarely of a catastrophic nature, but certainly they each cost time and/or money, the sum of which has an impact on both the project and the companies involved.

It becomes clear that reducing the time needed to produce sufficiently detailed visual representations and ensuring that they can be read and 
understood by non-engineers is crucial to the progression and increased efficiency of the market.

\section{SOLUTIONS}

The problems that exist with visualisations provide a significant challenge to any engineer, project team or company. These problems are unlikely to result in the immediate collapse of a project or a company, but the inefficiency and subsequent costs cannot, ultimately, be sustained.

The most obvious solution to the cost and time problems that any company can easily implement is to simplify the visualisation. However, this will not give the engineers the required information and over-simplifying can look un-professional, a serious problem in such a competitive market. It is therefore crucial that the right balance of time, cost and detail is struck.

The solution currently employed by engineers in the Assembly Automation and Test field is to make the majority of the conceptual level design decisions at a very early (pre-quote) stage. The sales team, which consists of experienced mechanical and electrical engineers and project managers, will perform the iterations between Sketches and Diagrams rapidly generating a list of potential solutions and then reducing this to a short list of 2-4 promising solutions. The sales team will then finalise their decision as to which of the concepts to quote on. Once this final decision has been made, the sales team will produce the Drawings that will provide the basis for both the quote and the handover of the project to the project team.

The communication between the individuals involved within the sales team is most often a combination of verbal and visual, with both Sketches and Diagrams being used. This process is constrained by time; it is essential that a quote of sufficient detail is produced quickly, both to minimise cost and to satisfy the potential customer. This results in the sales team presenting Drawings to the customer and, assuming a successful quote, to the project team. The project teams are responsible for the project beyond the quotation acceptance and ultimately implement the physical realisation of the design. The project team will then further develop the Drawings into Details, which are then used to produce the final system.

This method has proved to be successful in enabling projects to move swiftly and operate within budget. However, there are problems associated 
with this method; it constrains the project team in terms of design freedom and it also places an even greater pressure on the visualisations. The Sketches and Diagrams are needed to be efficient in communicating within the sales team, whilst also providing adequate "stand-alone" capability that they will be able to be used as archive information. The Drawings are under the most pressure as they must not only communicate the design to other engineers within the company, but also sell the idea to the customer.

The ideal solution, from an engineer's point of view, would be a tool that enables him or her to produce a Drawing, with the benefits of high detail and reduced requirement for additional information, but in the time-frame and ease of a Sketch. This is of course the ideal solution and would be a very ambitious target for any company or project. However, by gaining an understanding of how and why visualisations are used as well as developing and optimising a tool, perhaps even for each of the classes, progress towards this ultimate target can be made.

Achieving this is not straightforward. What must be accepted is that the solution cannot be provided by a single company trying to impose its ideas on the rest of industry. The solution must come from collaborative work, with representatives from each link in the supply chain and a mixture of both industry and academia involved. The most immediate problem with this strategy is that if you ask ten people what is "too complex" or "too simple" you will doubtless get ten different answers. This is not just due to the nonquantifiable nature of "complexity" but is also due to the fact that individual companies have their own standards and practices, which they will require any solution to fit to, not the other way around. Any solution must therefore be flexible, adaptable or customisable to specific user requirements.

The IMechE in the UK also recognise the problem and believe one of the most promising solution routes is in Simulation [4]. The aim of simulation is to produce highly impressive (aimed at non-engineer customers) and detailed (for the engineers in the companies) visualisations.

Another way of solving these problems, which also involves simulation, is to consider producing a library of equipment and assembly cell models, an idea being developed in the E-RACE project [5]. E-RACE utilises the Internet to provide the required user interface, which allows instant communication between any two companies. These models are both visual representations of the physical item and also contain the performance and costing figures. This concept is clearly in tune with current engineering 
practises; it is very rare, even for a bespoke manufacturer, to design and build an assembly automation machine from scratch. It is far more common to utilise a collection of "modules", either designed in-house or bought-in, and make the necessary adaptations to ensure that a working machine is produced.

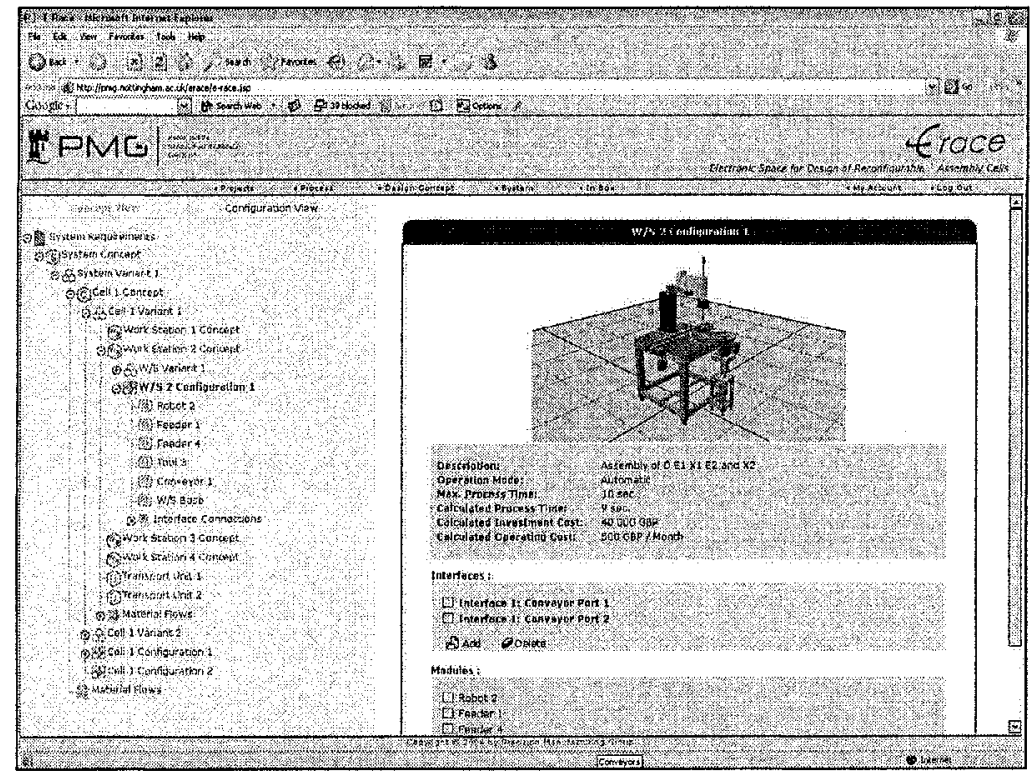

Figure 5. Screen shots from the E-RACE portal. Courtesy of www.e-race.org.uk.

The E-RACE project builds on this, adding a structured user requirement specification and quotation system. This concept can be further developed by designing the machinery to suit this design process. This is being investigated by the EUPASS project [6], which has links to E-RACE.

\section{CONCLUSIONS}

It is generally accepted that the Detail class of visualisation has been greatly improved by the almost universal use of CAD. Details are time consuming to produce by nature and necessity; they must enable those 
involved to convert the raw materials into the final product. Realistically, any further improvements to this class will be the result of software improvements by commercial companies.

It is in the other three classes, for which there is no specific or dedicated tool, that the big improvements can and should be made. Engineers may currently employ various tools, often a CAD tool or even simply MS Office program, to assist them with the earlier classifications, but these are compromises.

Ultimately, an engineer wants a tool or suite of tools that are dedicated to each stage of his or her visualisation needs. The optimum solution would be to combine the benefits of Sketches with those of Drawings and Details.

\section{REFERENCES}

1. Boden, D. (1994) The Business of Talk, $1^{\text {st }}$ Edition, Polity Press, London.

2. Koskinen, I. Plans, evaluation and accountability. Social Research Online, 2000, www.socresonline.org.uk

3. Chua, C K et al. (2003) Rapid Prototyping: Principles and Application, $2^{\text {nd }}$ Edition, World Scientific, New Jersey, NJ

4. Rooks, B. 2000, Winning Ways for Manufacturing, Assembly Automation, Vol 20, number 1, pp 35-39.

5. UK Eureka Consortium. E-Race, Eureka Project: Eureka Factory E!2851. www.erace.org.uk

6. EUPASS www.eupass.org 